\title{
Sub-arcsecond imaging of the radio continuum and neutral hydrogen in the Medusa merger
}

\author{
R. J. Beswick ${ }^{1}$, S. Aalto ${ }^{2}$, A. Pedlar ${ }^{1}$, and S. Hüttemeister ${ }^{3}$ \\ 1 The University of Manchester, Jodrell Bank Observatory, Macclesfield, Cheshire SK11 9DL, UK \\ e-mail: Robert.Beswick@manchester.ac.uk \\ 2 Onsala Rymdobservatorium, Chalmers Tekniska Högskola, 43992 Onsala, Sweden \\ e-mail: susanne@oso.chalmers.se \\ 3 Astronomisches Institut Ruhr Universität Bochum, Universitätßtr. 150, 44780 Bochum, Germany
}

Received 20 July 2005 / Accepted 24 August 2005

ABSTRACT

We present sub-arcsecond, Multi-Element Radio Linked Interferometer (MERLIN) observations of the decimetre radio continuum structure and neutral hydrogen (HI) absorption from the nuclear region of the starburst galaxy NGC 4194 (the Medusa Merger). The continuum structure of the central kiloparsec of the Medusa has been imaged, revealing a pair of compact radio components surrounded by more diffuse, weak radio emission. Using the constraints provided by these observations and those within the literature we conclude that the majority of this radio emission is related to the ongoing star-formation in this merger system.

With these observations we also trace deep $\mathrm{HI}$ absorption across the detected radio continuum structure. The absorbing HI gas structure exhibits large variations in column densities. The largest column densities are found toward the south of the nuclear radio continuum, co-spatial with both a nuclear dust lane and peaks in ${ }^{12} \mathrm{CO}(1 \rightarrow 0)$ emission. The dynamics of the $\mathrm{HI}$ absorption, which are consistent with lower resolution ${ }^{12} \mathrm{CO}$ emission observations, trace a shallow north-south velocity gradient of $\sim 320 \mathrm{~km} \mathrm{~s}^{-1} \mathrm{kpc}^{-1}$. This gradient is interpreted as part of a rotating gas structure within the nuclear region. The HI and $\mathrm{CO}$ velocity structure, in conjunction with the observed gas column densities and distribution, is further discussed in the context of the fuelling and gas physics of the ongoing starburst within the centre of this merger.

Key words. galaxies: individual: NGC 4194 - radio lines: galaxies - ISM: kinematics and dynamics - galaxies: ISM galaxies: starburst - radio lines: ISM

\section{Introduction}

The Medusa merger (NGC 4194) is a nearby $(D=39 \mathrm{Mpc})$ star-forming galaxy of intermediate infrared luminosity $\left(L_{\mathrm{FIR}}=\right.$ $\left.8.5 \times 10^{10} L_{\odot}\right)$. As such the Medusa merger falls into a luminosity class, considerably lower than the well-studied ultraluminous infrared galaxies (ULIRGs) but it still appears to show areas of intense starburst activity (Armus et al. 1990; Prestwich et al. 1994). The optical morphology of the Medusa merger, as its name suggests, traces clumpy and extended features which stretch $\sim 1^{\prime}$ to the north of the main optical nucleus. These "hair-like" extensions, indicative of tidal tail features resultant from the galaxy's ongoing merging event, contain significant volumes of molecular gas (Aalto et al. 2001) although no indications of ongoing star formation in the tidal tail have, as yet, been detected (Armus et al. 1990). The majority of star formation in the Medusa merger is concentrated in the extended nuclear starburst (Armus et al. 1990).

A detailed study of the molecular gas $(\mathrm{CO} J=1 \rightarrow 0)$ composition and kinematics in NGC 4194 has been previously made at arcsec angular resolutions at the Owens Valley Radio Observatory (OVRO) by Aalto \& Hüttemeister (2000, hereafter $\mathrm{AH} 00)$. This investigation revealed that the ${ }^{12} \mathrm{CO}$ emission is extended over an area of $25^{\prime \prime}(4.7 \mathrm{kpc})$ covering the central and north-eastern part of the optical galaxy. The $\mathrm{CO}$ emission also traces the two prominent dust lanes that cross the central region and extends into the northern tidal tail. The majority of the $\mathrm{CO}$ $(\sim 70 \%)$ is found with the central $2 \mathrm{kpc}$ of the galaxy, with $15 \%$ of this gas residing in a compact region 1".5 south of the radio nucleus.

We have used the UK's Multi-Element Radio Linked Interferometric Network (MERLIN) to observe both the $1.4 \mathrm{GHz}$ radio continuum and neutral hydrogen (HI) absorption structure of the central few hundred parsecs of NGC 4194 at sub-arcsec angular resolutions. This constitutes the highest angular resolution study made to date of both the radio continuum and neutral gas structure in this merger galaxy. In this paper we present the results of these observations and place them in context with other observations of the radio continuum, and neutral and molecular gas in NGC 4194. The primary goal of this work is to provide high linear resolution information about the neutral gas within the core region of this star-forming galaxy, enabling the composition and dynamics of the central region to be investigated. In conjunction with lower resolution data (e.g. AH00; Aalto et al. 2001) these observations can be 
used to more fully chart the gas dynamics and fuelling of this intermediate luminosity starburst galaxy.

This paper is organised as follows. The observations and the data reduction processes undertaken are described in detail in the next section. This is followed by the presentation of the radio continuum and $\mathrm{HI}$ absorption results. In the subsequent sections we will discuss, in detail, these observational results, initially drawing conclusions regarding the neutral hydrogen and radio continuum structure of the central 0.5 kiloparsec region of the Medusa and then by placing these results in the context of other observations of this source's gas content.

Throughout this paper we will assume a distance of $39 \mathrm{Mpc}$ to NGC $4194\left(H_{0}=75 \mathrm{~km} \mathrm{~s}^{-1} \mathrm{Mpc}^{-1}\right)$, implying that at the distance of the source $189 \mathrm{pc}$ subtends an angle of 1 second of arc.

\section{Observations and data reduction}

\subsection{MERLIN}

NGC 4194 was observed using the MERLIN array, including the 76-m Lovell Telescope (Thomasson 1986), on 2003 March 15 at $1408.66 \mathrm{MHz}$. NGC 4194 was observed for $\sim 18 \mathrm{~h}$ interspersed with regular observations of the nearby phase calibration source $1205+545$. Throughout the observation dual hands of circular polarisation were recorded over an $8-\mathrm{MHz}$ band which were correlated into 64 spectral channels, each of bandwidth $125 \mathrm{kHz}$ providing a velocity resolution of $\sim 26 \mathrm{~km} \mathrm{~s}^{-1}$ channel $^{-1}$, centred upon the rest velocity of NGC 4194, $2560 \mathrm{~km} \mathrm{~s}^{-1}$. A single observation of OQ208 was used to obtain passband solutions along with a $30 \mathrm{~min}$ integration on $3 \mathrm{C} 286$, which was assumed to have a flux density of $14.794 \mathrm{Jy}$ at $1408 \mathrm{MHz}$ (Baars et al. 1977) and was subsequently used to calibrate the flux density scale for all sources.

Initial editing and calibration of these NGC 4194 data were carried out using local MERLIN software at Jodrell Bank Observatory, prior to these data being read into the Astronomical Image Processing Software (AIPS). Within AIPS further editing and bandpass calibration were applied to the data set. Phase corrections derived from the phase calibrator source $1205+545$ were also applied to these data, along with further phase corrections derived from self-calibration upon the continuum of NGC 4194. The calibrated uv data set was Fourier-transformed to produce spectral line data cubes with various tapering and data weighting schemes used in order to produce images that were sensitive to diffuse large scale radio emission and high resolution images of the compact components. The line-free channels were combined to produce continuum images which were subsequently subtracted from the spectral-line cubes. These continuum images along with the continuum subtracted spectral-line cubes were deconvolved using a CLEAN based algorithm to remove the instrumental response (Högbom 1974). The cleaned continuum images and continuum-subtracted cubes were then recombined and further analysis of these data were undertaken using standard spectral line tasks within AIPS.

These MERLIN data have a shortest projected baseline of $\sim 13 \mathrm{~km}$ between the Tabley and Jodrell Bank telescopes and hence these data are not sensitive to extended components greater than a few arcsecs. The consequences of this data-set including few low-order spacings is a hole in the uv coverage toward the centre of the uv plane. This feature of sparsely filled interferometric arrays, such as MERLIN, causes an under-sampling of the low-order spacings and hence an insensitivity to regions of smooth, extended flux. This effect can result in a bowl-shaped reduction in the flux density toward extended sources (see, for example Wills et al. 1998). This not only reduces the flux density of radio continuum components derived from images but also causes the calculated HI absorption opacities to be over estimated due to the reduction in the value of the extended continuum emission. The total flux measured from these observations is $28 \mathrm{mJy}$ compared to a lower resolution (beam size of $45^{\prime \prime}$ ) VLA $1.4 \mathrm{GHz}$ flux of $91 \mathrm{mJy}$ (Condon et al. 1990). The distribution of the radio emission detected in this VLA image covers an area is approximately 4 times larger than the region shown in Fig. 1. This implies that these observations are spatially filtering out approximately two thirds of the $1.4 \mathrm{GHz}$ flux. If it is assumed that this missing flux is spread over a Gaussian with an area matching the component detected by Condon et al., the maximum correction to the observed MERLIN map that is derived is $0.05 \mathrm{mJy}$ at any single point in the image. In the case of the observations presented in this paper we have further tested for the significance of these missing low-order spacings by taking slices across the images in several directions. Although some evidence for a slight reduction in the flux density of the images produced was found, it has not been directly corrected for in the images and spectra present since the correction required was found to be significantly smaller than the channel based noise levels in these data.

\subsection{Hubble space telescope (HST)}

In order to afford a good comparison between the sub-arcsec radio observations presented in this paper and the optical emission from this galaxy archival HST observations have been acquired. These NGC 4194 data (U2E66Y01T) consisted of single $600 \mathrm{~s}$ integration using a broad-band $F 606 \mathrm{~W}$ filter (see Fig. 1). Initially these data were reduced using both STSDAS commands within IRAF and STARLINK software before being exported as a FITS file. The procedure of applying accurate astrometry to the HST field was achieved by assigning known coordinates, obtained from the US Naval Observatory star catalogue, to a number of stars spread throughout the WFPC-2 field. This procedure resulted in a linear transformation and rotation being applied to the HST image in-order to minimise the residual position errors of the known star positions. No shear was applied to the image during this process. It is estimated that the absolute positional accuracy of the aligned MERLIN and HST images is $<0.1$ arcsec.

\section{Results}

\subsection{The $1.4 \mathrm{GHz}$ radio continuum}

A uv-tapered $(800 \mathrm{k} \lambda) 1.4 \mathrm{GHz}$ image of the central region of NGC 4194, derived from the line-free channels, is shown 


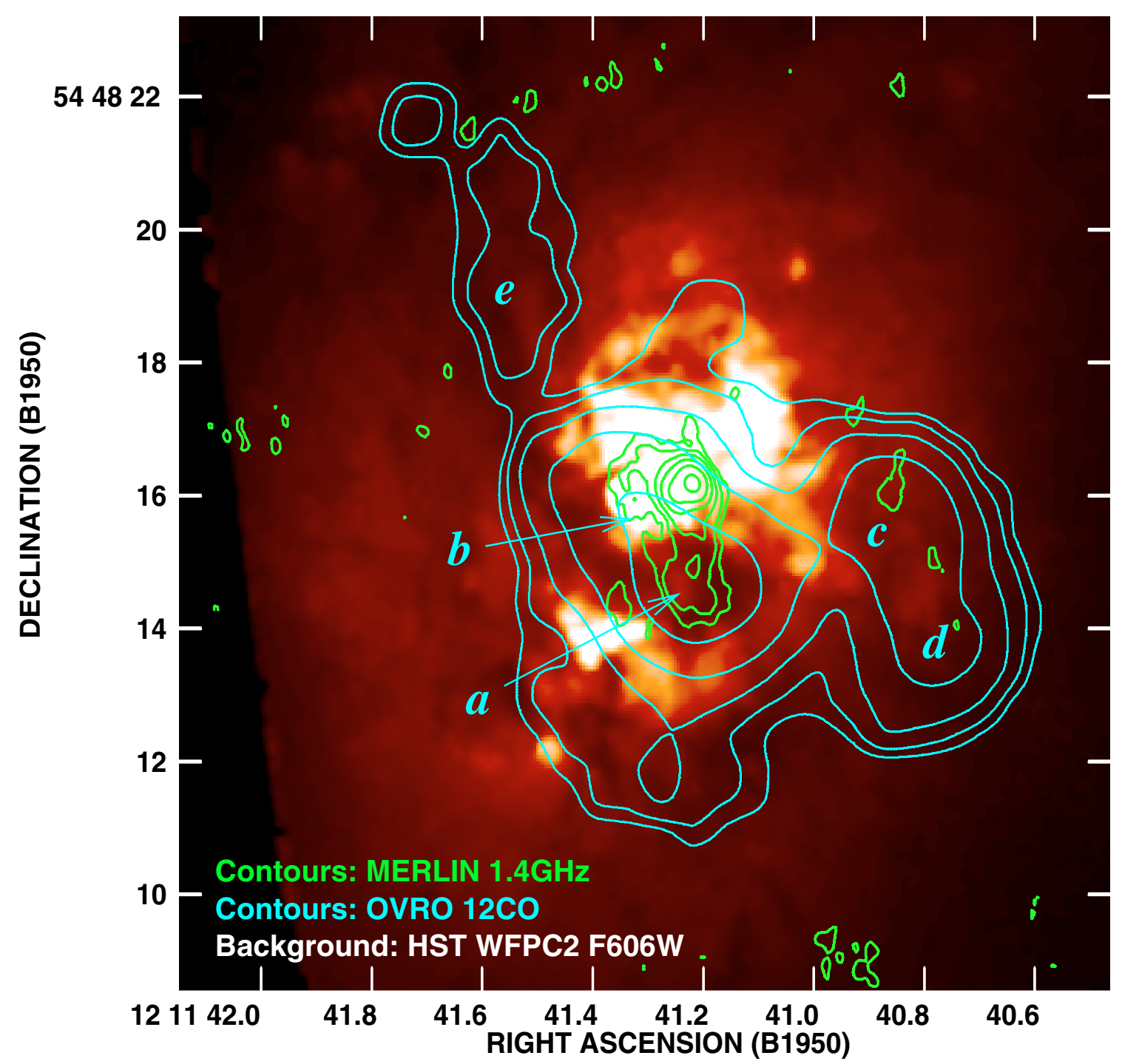

Fig. 1. Overlay of uv-tapered $(800 \mathrm{k} \lambda) 1.4 \mathrm{GHz}$ radio continuum emission in green contours and OVRO ${ }^{12} \mathrm{CO}$ (AH00) in pale blue contours over a F606W WFPC2 HST image. The contour levels on the radio continuum image are $-1,1,2,4,8,16$ and 32 times $0.3 \mathrm{mJy} \mathrm{bm}^{-1}$. The ${ }^{12} \mathrm{CO}$ contours are $1,2,4,8,16$ and 32 times $0.5 \mathrm{Jy} \mathrm{beam}^{-1} \mathrm{~km} \mathrm{~s}^{-1}$. The false-colour scale of the optical image is linear and arbitrary. The radio and $\mathrm{CO}$ images have synthesised beams of $0.446 \times 00^{\prime} 320$ and $22^{\prime \prime} 06 \times 11^{\prime \prime} 70$ respectively. The astrometrical alignment of the HST image with the other two images has an rms error of $\sim 100$ mas and is described in Sect. 2.2. Labels $a, b, c, d$ and $e$ indicate the ${ }^{12} \mathrm{CO}$ peaks as observed by AHOO.

as contours in Fig. 1, overlayed upon a false-colour archival WFPC2 HST image. A uv-taper was applied to these data in order to produce images which are as sensitive as possible to the weak and diffuse extended radio emission. A measured noise level of $0.1 \mathrm{mJy} \mathrm{bm}^{-1}$ has been determined for Fig. 1 and the image has an angular resolution of $0 ., 45 \times 0.32$. Figure 2 shows a high angular resolution, uniformly weighted, contour image of the core region of the galaxy. This figure has an angular resolution of $0{ }^{\prime} 17 \times 00^{\prime} 15$ and a noise level of $\sim 0.1 \mathrm{mJy} \mathrm{bm}^{-1}$. Table 1 lists the $1.4 \mathrm{GHz}$ flux densities and Gaussian fitted sizes of the two, bright, compact components seen in the uniformly weighted image (Fig. 2). The radio structure of the central few hundred parsecs of NGC 4194 is dominated by a pair of compact radio components (Fig. 2). These two components are separated by $\sim 0$ ' $^{\prime} 35(\sim 65 \mathrm{pc})$ along a PA of $120 \pm 5^{\circ}$. In the uv-tapered image shown in Fig. 1, and enlarged as the central image of Fig. 3, it can be seen that these two compact radio components are only partially resolved and are embedded in a halo of diffuse radio continuum which extends in a southerly direction.

\subsection{Neutral hydrogen absorption}

As can be seen in Fig. 3, extensive HI absorption is detected against the majority of the background radio continuum in the uv-tapered MERLIN image. Similarly HI absorption is also found against the core region in spectral line cubes made with uniform weighting. The HI absorption properties derived from the uv-tapered spectral line cube are listed in Table 2. These properties include the central positions of the spectra shown in Fig. 3, in addition to the Gaussian fitted velocities and widths of the absorption lines. Table 2 also lists derived 


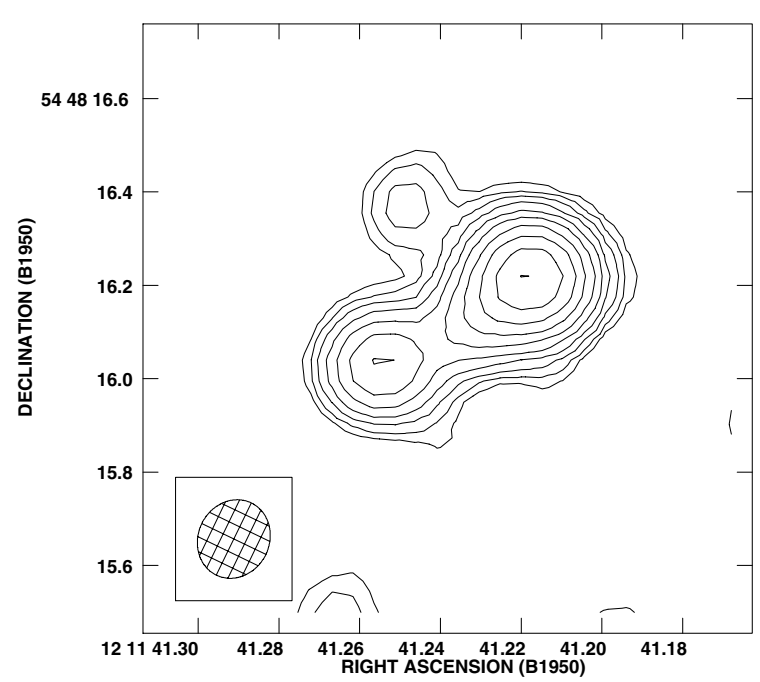

Fig. 2. Uniform weighted MERLIN $1.4 \mathrm{GHz}$ radio continuum image of the core of the Medusa merger with a synthesised restoring beam of $0.17 \times 0 .{ }^{\prime} 15$. Contour levels start at $0.35 \mathrm{mJy} \mathrm{bm}^{-1}$ and increase by factors of $\sqrt{2}$.

properties of these absorption lines, such as their peak opacities and column densities of HI along these lines of sight. The column densities listed have been calculated assuming that $N_{\mathrm{H}}=1.823 \times 10^{18} T_{\text {spin }} \int \tau \mathrm{d} V$. Although $T_{\text {spin }}$ can be reasonably assumed to be $100 \mathrm{~K}$ (Maloney et al. 1996) it should be noted that the value of $T_{\text {spin }}$ is dependent upon the physical conditions of the gas and will therefore vary significantly especially in the extreme environment of a nuclear starburst. These considerations will be discussed in more detail in a latter section.

In Fig. 4 we present three grey-scale images of the centre of the Medusa presenting the HI absorption detected against the radio continuum. The left-most (Fig. 4a) of these panels shows a grey-scale map of the HI absorption toward areas with significant radio continuum background. As can be seen in this image the line signal varies, with the absorption deepest toward the eastern edge of the brightest radio component. The map of integrated absorption shown in Fig. 4a does not represent the true HI column density since it is dependent upon the background continuum strength. In the central panel (Fig. 4b) the distribution of the integrated HI opacity is shown. This panel shows the distribution of neutral hydrogen and is directly proportional to the HI column density. It should, however, be noted that the process of deriving maps of optical depth involves the division of spectral line data-cubes with their associated radio continuum images, resulting in the opacity images produced being susceptible to errors in areas where the radio continuum is small (i.e. toward the edges of the image). Measures to minimise this effect have been made, although some unphysical values in a few pixels toward the edges of the image may remain. However considering this factor the variations in opacity seen are significantly larger than the errors in the image. Plus the values and features seen in this image are consistent with those observed and calculated from the absorption spectra (see Fig. 3) and absorption maps (Fig. 4a). The HI integrated opacity shows an increase in the column densities south of the radio continuum peak which is consistent with the positions of the peaks of the $\mathrm{CO}$ emission observed by AHOO (labelled in Figs. 1 and 4a).

Figure $4 \mathrm{c}$ shows that the velocity structure of the HI follows a distinct north to south velocity gradient on the largest scales along with an $\mathrm{N}-\mathrm{W}$ to $\mathrm{S}-\mathrm{E}$ velocity gradient across the two bright, compact radio components. Figure 5 shows a position (Declination) verses velocity contour plot of the absorption averaged over the entire RA extent of the radio continuum source. This figure complements the two dimensional velocity distribution shown in Fig. 4c by showing in detail the north to south velocity gradient in the source. As can be seen from these figures the velocity structure illustrated in Fig. $4 \mathrm{c}$ traces a gradient of $\sim 60 \mathrm{~km} \mathrm{~s}^{-1} \operatorname{arcsec}^{-1}$.

\section{Discussion}

\subsection{High resolution radio continuum structure of the centre of the Medusa}

In the highest angular resolution $\left(0{ }^{\prime} 17 \times 0{ }^{\prime} 15\right)$ image produced using these MERLIN data (Fig. 2) the majority of the radio structure of the Medusa galaxy is resolved away leaving a pair of compact nuclear components (see Table 1 and Fig. 2) with a small, weak extension toward the north-east of the brightest component. This double structure is reminiscent of the core regions of many low powered Seyfert galaxies (e.g. Thean et al. 2000). However, the lower angular resolution uvtapered images show these components to be sitting in an extensive, diffuse, radio emission toward the south of these compact components (see Figs. 1 and 3). These uv-tapered images (Figs. 1 and 3) are consistent with other studies of this source previously made at higher frequencies using the Very Large Array (VLA) (e.g. Ulvestad et al. 1981). Figure 3 is still insensitive to many of the weak, larger scale features observed by Ulvestad et al. (1981). More recent multi-frequency (5 to $15 \mathrm{GHz}$ ) VLA observations (S. Neff, private comm.; Neff et al. 2001) show that in addition to the compact components and diffuse emission observed in these MERLIN $1.4 \mathrm{GHz}$ data more extended emission is present. In particular, in deep $5 \mathrm{GHz}$ VLA images (S. Neff, private comm.), an "arm-like" extension arcing from the north of the nuclear region and ending approximately 4 arcsec to the west of this region in a strong compact component, is observed. This feature is also seen in VLA images published by Ulvestad et al. (1981). Although some traces of this "arm-like" emission can be seen in our uv-tapered images (see Fig. 1) the majority of these features are not detected by our observations. Neff et al. (2001) also report $\sim 50$ compact sources $\left(>5 \times 10^{18} \mathrm{~W} \mathrm{~Hz}^{-1}\right)$. Of the brightest of these compact sources $\sim 50 \%$ nominally have flat spectral indices and maybe associated with thermal radio emission from HII regions, whereas the remainder have steeper spectral indices that are indicative of emission dominated by non-thermal mechanism (such as supernovae remnants or AGN). Our sub-arcsec 1.4 GHz observations are, as mentioned above, insensitive to much of the diffuse extended radio emission detected with the VLA, but more sensitive to emission from synchrotron sources than thermal sources; the combination of these effects and our 
Table 1. The $1.4 \mathrm{GHz}$ radio continuum properties of the core components ${ }^{a}$.

\begin{tabular}{lllllll}
\hline \hline $\begin{array}{l}\text { RA (B1950) } \\
12^{\mathrm{h}} 11^{\mathrm{m}}\end{array}$ & $\begin{array}{l}\text { Dec (B1950) } \\
54^{\circ} 48^{\prime}\end{array}$ & $\begin{array}{l}\text { Peak } \\
\text { flux density } \\
\left.(\mathrm{mJy} \mathrm{bm})^{-1}\right)\end{array}$ & $\begin{array}{l}\text { Integrated } \\
\text { flux density } \\
(\mathrm{mJy})\end{array}$ & Angular size & $\begin{array}{l}\text { Linear size } \\
(\mathrm{pc})\end{array}$ & PA \\
\hline $41^{\mathrm{s}} 22$ & $16^{\prime \prime} 210$ & 8.20 & 12.6 & $00^{\prime} 140 \times 00^{\prime} 093$ & $27.8 \times 17.6$ & 97.9 \\
$41^{\mathrm{s}} .25$ & $16^{\prime \prime} 038$ & 2.84 & 5.11 & $00^{\prime} 187 \times 00^{\prime} 097$ & $35.3 \times 18.3$ & 106.6 \\
\hline
\end{tabular}

\begin{abstract}
${ }^{a}$ Listed properties are for the two compact nuclear components. Flux densities and positions have been derived from Gaussian fits to our uniformly weighted image (Fig. 2).
\end{abstract}

sensitivity levels accounts for the differences in observed emission structures.

\subsection{The neutral hydrogen absorption}

High optical depth $\left(\tau_{\text {peak }}>0.5\right)$ neutral hydrogen absorption is detected against almost all of the radio continuum emission detected (Fig. 1). The absorbing HI gas is observed to show significant variations in opacity across the radio continuum source. The HI column densities observed against the radio continuum emission are consistently higher toward the southern half of the nuclear radio continuum. These variations can be seen in both the maps of the integrated HI absorption and opacity (Figs. 4a,b) as well as in the individual spectra (Fig. 3 and Table 2). This implies that the HI observed, is not part of a uniform homogeneous screen of neutral foreground gas. It is likely that the large scale variations in the HI opacities observed are the result of high gas column densities along the lines of sight of other large scale features, such as the dust lanes. Whereas smaller, more localised, variations seen in Fig. 4b (on size scales of order a beam $[\sim 50-60 \mathrm{pc}])$ are likely to be due to single, or the superposition of several, gas clouds along the sight line. In Fig. 1 the distribution of the radio continuum relative to the WFPC2 $R$-band image shows a thick dust lane arc that crosses the southern part of the MERLIN radio continuum in an approximately east-west direction. The position of this dust lane, which is also co-spatial with the $\mathrm{CO}$ emission (red contours in Fig. 1; AH00), is coincident with the area of highest opacity $\mathrm{HI}$ absorption. It is also reasonable to assume that the gas, both $\mathrm{CO}$ and $\mathrm{HI}$, is associated with this dust lane (the relative distributions of these constituents is discussed in more detail in Sect. 4.4.1). Hence this implies that the radio continuum originates from deeper within the source than these components or that these elements of the neutral ISM and the radio emitting material are mixed resulting in these observations underestimating the true HI column densities.

The HI velocity field shown in Fig. 4c traces a dominant but shallow velocity gradient with a north to south direction. This gradient is also shown in Fig. 5. In this averaged positionvelocity slice the broad absorption tracks a velocity gradient of $\sim 60 \mathrm{~km} \mathrm{~s}^{-1} \operatorname{arcsec}^{-1}\left(\sim 320 \mathrm{~km} \mathrm{~s}^{-1} \mathrm{kpc}^{-1}\right)$. This velocity gradient extends over $\sim 2$ '. 5 ( $470 \mathrm{pc}$ ) of the background radio continuum source. The north-south orientation of the velocity field is consistent with high resolution $\mathrm{CO}$ observations from the same region (AH00). The details of the velocity field (Fig. 4c) show that the primary gradient deviates from a north to south direction against the brighter northern radio continuum components. Hints of this directional change in the HI velocity gradient are visible in the highest angular resolution $\left(\sim 1^{\prime \prime} .7\right) \mathrm{CO}$ data presented by AHOO.

\subsection{The source of the radio emission in the Medusa}

Although the Medusa merger is an order of magnitude fainter at infrared wavelengths than the well studied ULIRG galaxies, such as Arp 220, it has in the recent past undergone, and continues to undergo, significant star-formation activity. This activity has presumably been triggered by its advanced merger state. However, it is also widely accepted that gas rich mergers, such as NGC 4194, can also host AGN activity in the remnant nuclei of the merged sources (Genzel et al. 1998). This phenomenon is observed in some composite starburst-AGN sources (e.g. Beswick et al. 2001; Gallimore \& Beswick 2004; Kewley et al. 2000) and is also evident in some minor mergers which host more powerful AGN such as radio galaxies (Heckman et al. 1986). As discussed previously the radio continuum observed from the Medusa merger, at $1.4 \mathrm{GHz}$, traces both the areas of starburst activity, as well the more compact radio components that may be AGN related. In the rest of this section we will discuss both the nuclear starburst of the Medusa and its rate of star-formation, as well as briefly explore the possible evidence that a weak AGN exist in the core of this galaxy.

\subsubsection{An AGN component?}

NGC 4194 is optically classified as a peculiar, blue compact galaxy with a strong HII emission line spectra and a blue continuum indicative of a young stellar population (Liu \& Kennicutt 1995). However, as discussed above the two compact radio components imaged in the centre of this galaxy show a structure that is similar to that observed in many low luminosity Seyfert galaxies. These two components are relatively low in luminosity, $5.48 \times 10^{20}$ and $2.22 \times 10^{20} \mathrm{~W} \mathrm{~Hz}^{-1}$, but are compact on MERLIN scales $(<25 \mathrm{pc})$, implying that the strongest of these components has a brightness temperature in excess of $4.5 \times 10^{4} \mathrm{~K}$. Searches for an AGN-like radio core using VLBI techniques in the Medusa have so far not detected any bright compact radio components (Lonsdale et al. 1992; van Breugel et al. 1981).

In addition to this continuum information these spectral line observations, along with those of $\mathrm{AHOO}$ provide information regarding the gas velocity distribution within the central region 


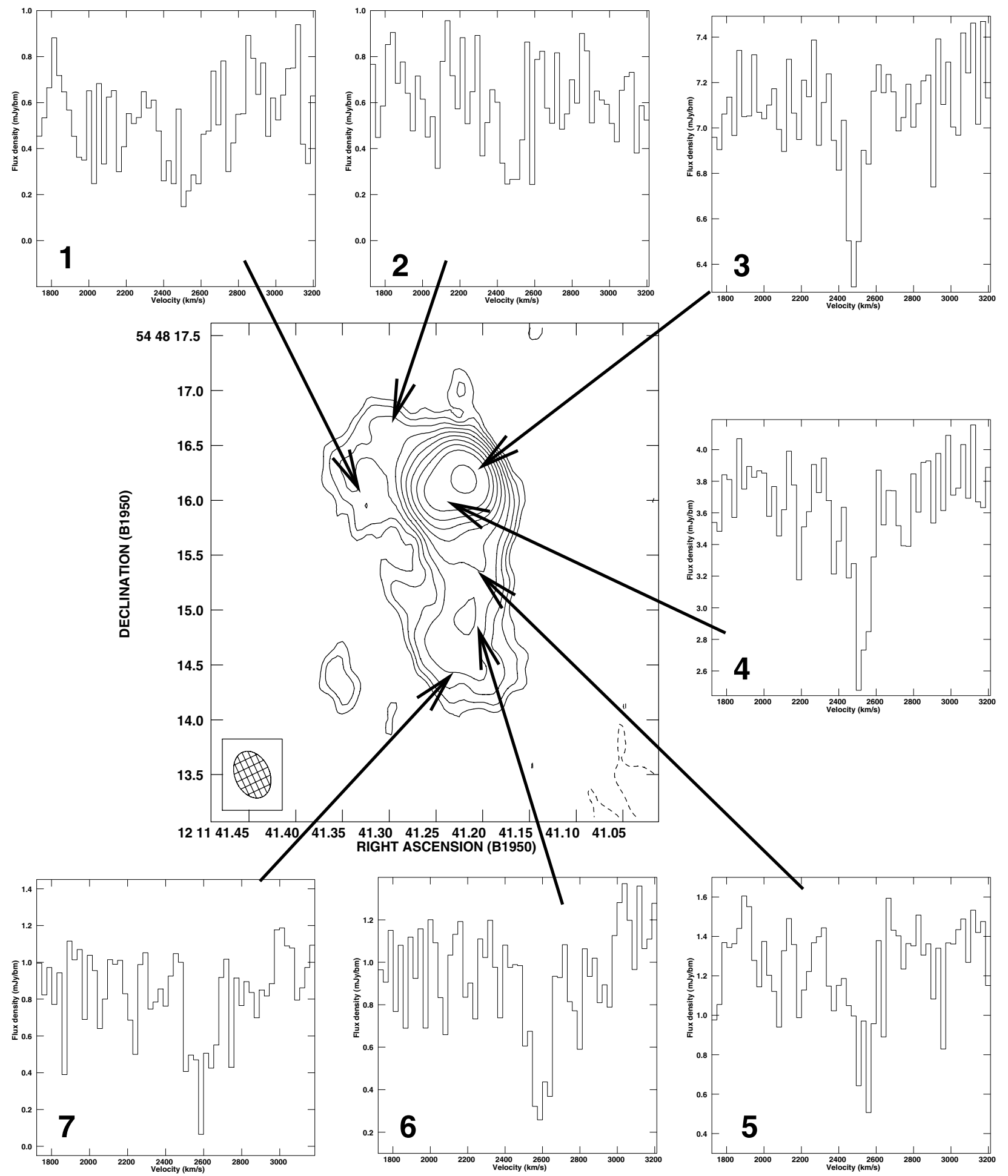

Fig. 3. Montage of 800-k $\lambda$ uv-tapered radio continuum structure and HI absorption spectra. Contour levels of the central image start at $0.3 \mathrm{mJy} \mathrm{bm}^{-1}$ and increase by factors of $\sqrt{2}$ with an angular resolution of $0{ }^{\prime} .45 \times 0$ ' 32 .

of NGC 4194 which can be used to place some limits on the mass enclosed within the central few arcsec. From these HI observations we observe a velocity gradient in the neutral gas of $\sim 320 \mathrm{~km} \mathrm{~s}^{-1} \mathrm{kpc}^{-1}$ over the central 2 '.5 (470 pc) which, if assumed to be in circular motion, implies a limit on the enclosed mass of $\sim 10^{6} M_{\odot}$. Such a limit constrains the mass of any black hole within the centre of the NGC 4194.
However, considering the limits on the luminosities and sizes of these radio continuum components, the presence or not, of a weak AGN located at the position of one of these two components cannot be categorically ascertained. The evidence from the VLBI studies of Lonsdale et al. (1992) show that no high brightness temperature synchrotron $>1.8 \mathrm{mJy}$ at $\lambda=18 \mathrm{~cm}$ is detected. This supports the case for little or no 
Table 2. The HI absorption properties of the central region of the Medusa merger.

\begin{tabular}{|c|c|c|c|c|c|c|}
\hline Label $^{a}$ & $\begin{array}{l}\mathrm{RA}(\mathrm{B} 1950)^{a} \\
12^{\mathrm{h}} 11^{\mathrm{m}}\end{array}$ & $\begin{array}{l}\operatorname{Dec}(\mathrm{B} 1950)^{a} \\
54^{\circ} 48^{\prime}\end{array}$ & $V_{\mathrm{H}}\left(\mathrm{km} \mathrm{s}^{-1}\right)$ & $\Delta V\left(\mathrm{~km} \mathrm{~s}^{-1}\right)(\sigma)$ & $\tau_{\text {peak }}$ & $\begin{array}{l}N_{\mathrm{H}}^{\mathrm{c}} \times 10^{19} T_{\text {spin }} \\
(\text { atoms cm } \\
-2\end{array}$ \\
\hline $1^{b}$ & $41^{\mathrm{s}} .32$ & $166^{\prime \prime} 085$ & - & - & - & - \\
\hline $2^{b}$ & $41^{\mathrm{s}} .31$ & $16 . " 545$ & - & - & - & - \\
\hline 3 & $41^{\mathrm{s}} .22$ & $16 . " 206$ & 2482 & 41 & 0.115 & 0.08 \\
\hline 4 & $41^{\mathrm{s}} .25$ & $16 ! .059$ & 2520 & 49 & 0.533 & 2.02 \\
\hline 5 & $41^{\mathrm{s}} .23$ & $155^{\prime \prime} 635$ & 2528 & 58 & 0.964 & 11.27 \\
\hline 6 & $41^{\mathrm{s}} .22$ & $144^{\prime \prime} 915$ & 2587 & 42 & 1.350 & 4.82 \\
\hline 7 & $41^{\mathrm{s}} .25$ & $144^{\prime \prime} 600$ & 2586 & 52 & 1.425 & 5.89 \\
\hline
\end{tabular}

${ }^{a}$ Labels and positions listed refer to the centre of the areas over which the spectra in Fig. 3 are averaged.

${ }^{b}$ No limits upon the optical depth are given in areas where the radio continuum flux density is too low to provide a physically important limit.

${ }^{c}$ Column densities have been calculated assuming $N_{\mathrm{H}}=1.823 \times 10^{18} T_{\text {spin }} \int \tau \mathrm{d} V$. These are derived from the absorption opacities and line widths over areas of approximately a beam in size. The value of $T_{\text {spin }}$ is assumed for the purposes of the discussion to be $100 \mathrm{~K}$, however this variable will be discussed in more detail in Sect. 4.4.3.

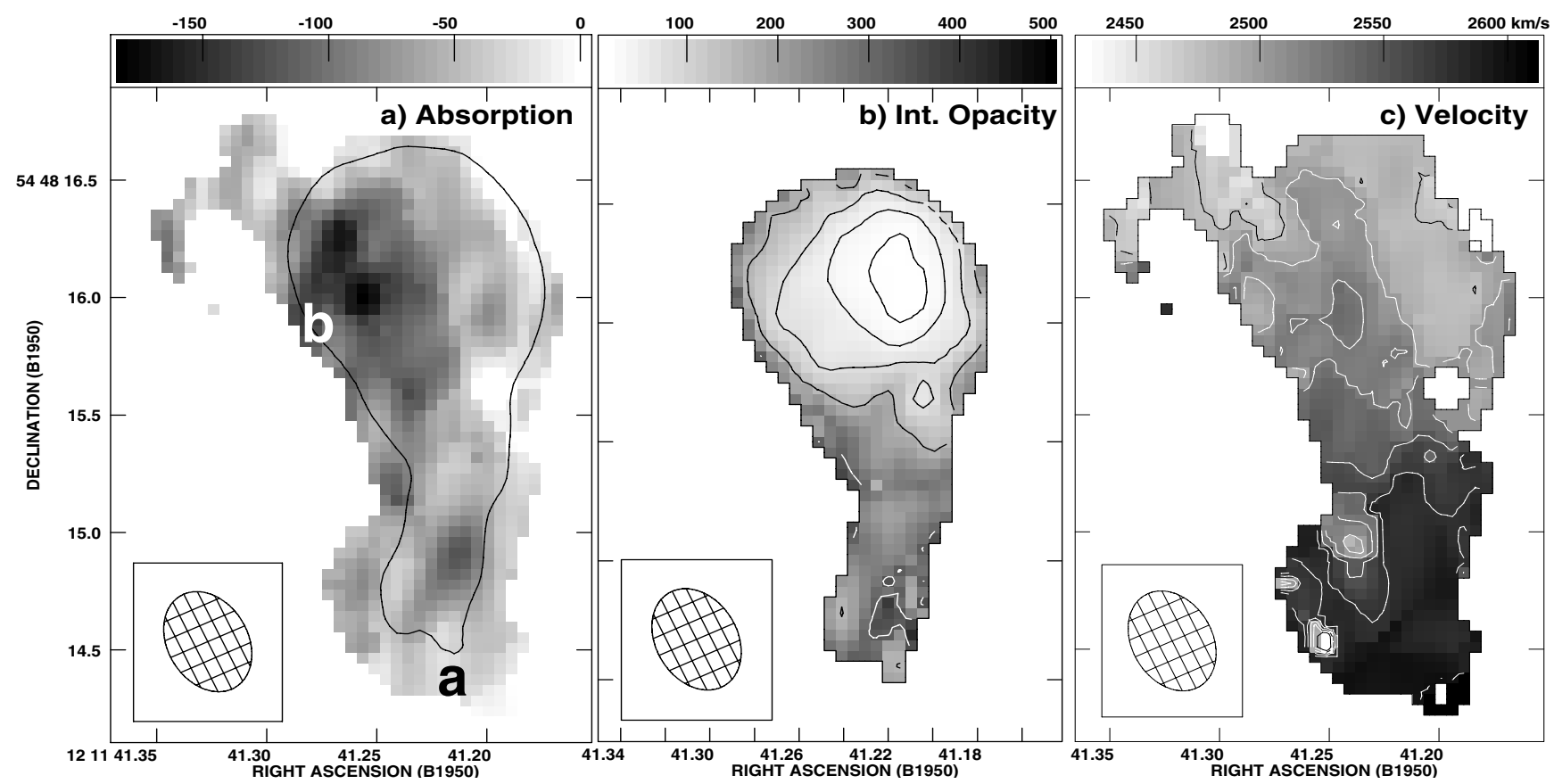

Fig. 4. a) The (leftmost) image shows the integrated HI absorption signal across the centre of the Medusa merger. This has been derived from the continuum subtracted spectral line cube. The grey-scale flux density range is $0 \rightarrow-185 \mathrm{mJy} \mathrm{bm}^{-1} \mathrm{~km} \mathrm{~s}^{-1}$. Note the absorption shown is dependent on the strength of the continuum emission. The two symbols ( $a$ and $b$ ) shown on the left-most image represent two of the CO emission peaks from AH00. A single contour $1 \mathrm{mJy} \mathrm{bm}^{-1}$ radio continuum contour is overlayed. Areas external to this contour have the lowest levels of radio continuum and have been excluded in the derivation of Fig. $4 \mathrm{~b}$. b) The (central) image shows the integrated optical depth $\left(\int \tau \mathrm{d} V\right.$ ) across the same region as a). The grey-scale range shown is $15 \rightarrow 500$ and the contour levels are 10, 20, 40, 80, 160, 320 and 640 times 100 . It should be noted that errors in this image increase in areas where the radio continuum flux density is at a minimum. This effect results in some erroneous values toward the edges of the image, in order to remove the most severe of these effects this image has been blanked in areas where the radio continuum is less than $1 \mathrm{mJy} \mathrm{beam}^{-1}$. c) The (right-hand) map represents the HI absorption velocity field over areas where significant radio continuum and absorption line signal were detected. The grey scale range of the image is $2430 \rightarrow 2610 \mathrm{~km} \mathrm{~s}^{-1}$, the overlayed contours have increments of $25 \mathrm{~km} \mathrm{~s}^{-1}$ between $2475 \rightarrow 2650 \mathrm{~km} \mathrm{~s}^{-1}$.

AGN related radio emission. Although it should be noted that southern nucleus of the binary AGN NGC 6240 contains a very weak compact VLBA core with flux of $\approx 1 \mathrm{mJy}$ (Gallimore \& Beswick 2004) which is free-free absorbed at $18 \mathrm{~cm}$. Further high resolution multi-frequency radio imaging and X-ray observations are required to investigate the possible scenario that there is an AGN buried in the centre of this source.

\subsubsection{The starburst and star-formation rate}

As has already been stated the Medusa merger is dominated, in terms of its optical and infrared emission by starburst activity. Starburst emission results in relativistic charged particles accelerating in supernovae remnants (SNRs) and radio supernovae (RSNe), which produces the radio emission, such as 


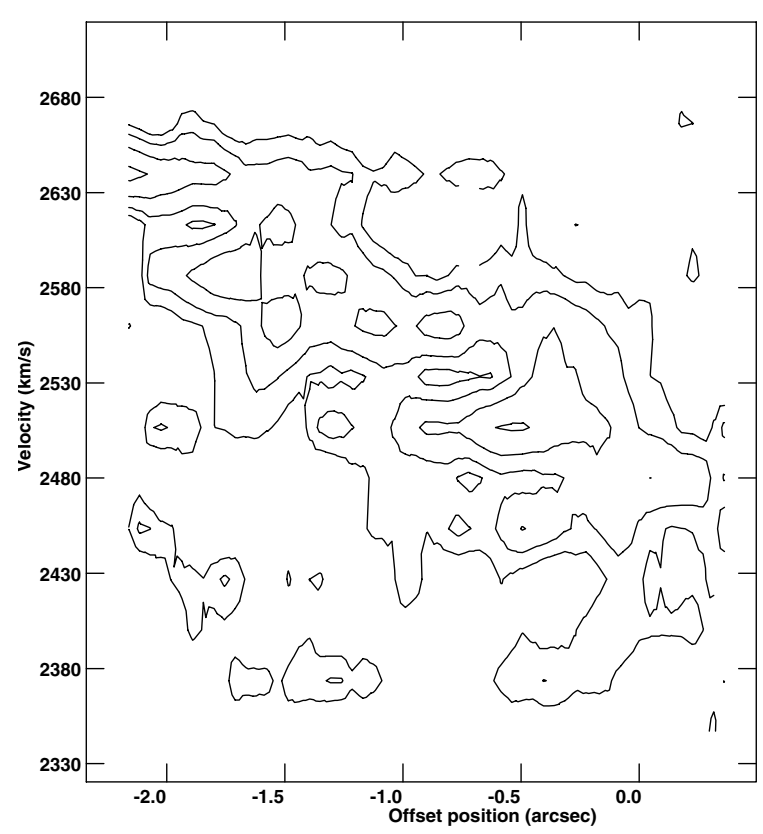

Fig. 5. A position velocity diagram of $\mathrm{HI}$ absorption in the central region of the Medusa merger. The absorption has been averaged over the entire RA range of the radio source, where the radio continuum is in excess of $0.6 \mathrm{mJy}$ beam $^{-1}$. The contour levels are set at $-8,-5.657$, $-4,-2.282,-2,-1.414,-1$ and 1 times $0.36 \mathrm{mJy}$ beam $^{-1}$. The position axis is declination relative to $54^{\circ} 48^{\prime} 16^{\prime \prime} 4$.

found in other nearby starburst galaxies (e.g. M 82 - Muxlow et al. 1994).

It has been shown, for example by Condon (1992) and Cram et al. (1998), that the $1.4 \mathrm{GHz}$ radio emission from star forming galaxies can be considered as a direct tracer for the supernovae rate. If this is assumed, the star-formation rate (SFR) of the source can also be estimated, albeit with an initial mass function (IMF) adjusted to produce massive enough stars to form supernovae that result in significant radio emission (i.e. $M \geq 8 M_{\odot}$ ) such that,

$S F R_{1.4}=5.5 \times \frac{L_{1.4}}{4.6 \times 10^{21} \mathrm{~W} \mathrm{~Hz}^{-1}} M_{\odot} \mathrm{yr}^{-1}$

(e.g. Haarsma et al. 2000; Hopkins et al. 2002). In this equation the multiplying factor of 5.5 is included to convert the mass range $5-100 M_{\odot}$ used by Condon (1992) to the range 0.1-100 $M_{\odot}$, assuming a Salpeter IMF. The low angular resolution (arcsecond), 1.4 GHz VLA flux of NGC 4194 is $92.7 \mathrm{mJy}$ (Condon et al. 1990), implies a total $S F R_{1.4} \approx 20 M_{\odot} \mathrm{yr}^{-1}$. In the case of the Medusa, and most starburst and ULIRG systems, the accuracy of the SFR as derived from optical data (e.g. H $\alpha$ observations) will be severely affected by the large and variable nature of the obscuration in this source. Using $\mathrm{H} \alpha$ observations Storchi-Bergmann et al. (1994) quote a $S F R_{\mathrm{H} \alpha} \approx 38 M_{\odot} \mathrm{yr}^{-1}$ (assuming $H_{0}=50 \mathrm{~km} \mathrm{~s}^{-1} \mathrm{Mpc}^{-1}$ ) which corresponds to a more modest value of $\sim 17 M_{\odot} \mathrm{yr}^{-1}$, if $H_{0}=75 \mathrm{~km} \mathrm{~s}^{-1} \mathrm{Mpc}^{-1}$ is assumed. This value shows a remarkable consistency with the $S F R_{1.4}$ value obtained from the total radio flux considering the uncertainties that are introduced by the high levels of nuclear obscuration within NGC 4194.
In our higher resolution radio data only the emission from the intense, compact nuclear star-forming regions can be detected. Applying equation 1 to our total MERLIN flux of 28 mJy implies a $S F R$ of $\sim 6 M_{\odot} \mathrm{yr}^{-1}$ for the Medusa; compared to the far-infrared derived $S F R_{\text {fir }}=6-7 M_{\odot} \mathrm{yr}^{-1}(\mathrm{AH} 00)$. Clearly in the case of this radio determination the value can only be considered as a lower limit for the total SFR, since it only accounts for star-formation in the compact nuclear region.

Compact clumps of star-formation have been imaged in NGC 4194 by Weistrop et al. (2004) at UV wavelengths using the HST. They find that there are $\sim 40$ knots within the centre of NGC 4194. From their analysis they conclude that the total SFR within the youngest ( $<20 \mathrm{Myr}$ ) of these knots, which are primarily located in the nuclear region in areas broadly consistent with the distribution of the $1.4 \mathrm{GHz}$ MERLIN radio continuum, is $\sim 6 M_{\odot} \mathrm{yr}^{-1}$. This is consistent with our radio and far-infrared determinations of the SFR in the nuclear region.

The structure and luminosity of the radio emission in the lower resolution $1.4 \mathrm{GHz}$ radio images (Fig. 3) can easily be reproduced via pure starburst mechanisms with only a relatively modest nuclear SFR. In this scenario there is no requirement for a compact radio AGN although it is not precluded.

\subsection{The ISM in the nucleus of the Medusa}

\subsubsection{Distribution of $\mathrm{HI}, \mathrm{CO}$ and dust}

Using these absorption data only gas in front of the radio continuum can be detected. However it is also apparent the HI distribution throughout the nuclear region is not an even screen of gas. There are significant variation in the measured opacity of the absorbing HI (assuming a constant $T_{\text {spin }}{ }^{1}$ ), with high columns of gas situated toward the southern part of the radio emission. This area of increased HI column density is spatially coincident with the increased optical extinction from dust that arises from the cross-nuclear dust lane (see Fig. 1). The implication of this is that, in addition to the optical emission, the radio emission is situated behind the dust lane and that it is probable that the obscuring dust and neutral gas are physically associated. This is commonly seen in many nearby active galaxies with nuclear dust lanes (e.g. Cole et al. 1998; Beswick et al. 2002, 2003, 2004; Jackson et al. 2003).

If we assume a standard Galactic gas-to-dust ratio (Stavely-Smith \& Davies 1987), an inferred optical extinction can be derived from the HI column density. Assuming these ratios the optical extinction at $B$-band is $A_{B}=$ $8.62 \times 10^{-22} N_{\mathrm{H}}$ mag. Consequently a column density of $\sim 5 \times$ $10^{21} \frac{T_{\text {spin }}}{100 \mathrm{~K}}$ atoms cm $\mathrm{cm}^{-2}$ implies an extinction along the same line of sight as the dust lane (positions 5, 6 and 7), of $A_{B} \approx 4-5 \mathrm{mag}$, assuming constant $T_{\text {spin }} \approx 100 \mathrm{~K}$. This value is approximately 50 times larger than the implied levels of extinction against the compact radio components toward the north of this radio source which are not crossed by the nuclear dust lane (see Fig. 1).

AH00 mapped the distribution of $\mathrm{CO}(J=1 \rightarrow 0)$ in the nucleus of NGC 4194 at high angular resolution using OVRO.

\footnotetext{
1 Variations in the value of $T_{\text {spin }}$ across the radio continuum will also effect the line strength (see Sect. 4.4.2).
} 
In this study the $\mathrm{CO}$ emission in the velocity range $2506 \rightarrow$ $2570 \mathrm{~km} \mathrm{~s}^{-1}$ (see Fig. 7 of AH00) is also found to trace the primary dust lane of the Medusa. AH00 observed CO emission to have linewidths of $F W H M \sim 150 \mathrm{~km} \mathrm{~s}^{-1}$, larger but still comparable to those observed here (see Table 2). This is quite remarkable considering the angular resolution of these HI observations is an order of magnitude higher than the $\mathrm{CO}$ emission measurements. The consistency of these two linewidth measurements could imply that these two gas components, situated along the same line of sight, are well mixed over a range of angular scales $(\sim 2 \rightarrow 0.2$ arcsec $)$.

At the areas of strongest $\mathrm{CO}$ emission ( $\mathrm{a}$ and $\mathrm{b}$ in Fig. 1 and $\mathrm{AH} 00)$ the $\mathrm{CO}$ gas surface density is $\sim 500-1000 \mathrm{M}_{\odot} \mathrm{pc}^{-2}$ $\left(\sim 4-8 \times 10^{21}\right.$ molecules $\left.\mathrm{cm}^{-2}\right)$. These columns compare to the HI absorbing column densities of $\sim 6-12 \times 10^{21}$ atoms $\mathrm{cm}^{-2}$, assuming $T_{\text {spin }}=100 \mathrm{~K}$. These HI columns are merely a lower limit since they are only sensitive to the gas in front of the radio continuum. As stated above these observations also show an increased column density of HI toward the radio continuum situated along the line of sight of the dust lane, which is also coincident with the $\mathrm{CO}$, in particular $\mathrm{CO}$ emission peaks a (AH00 and Figs. 1 and 4). Thus it implies that the nuclear $\mathrm{CO}$, $\mathrm{HI}$ and dust are all situated along the same sight line.

\subsubsection{Dynamics of the cold ISM: the fuel for the evolving starburst}

Figures $4 \mathrm{c}$ and 5 show the velocity field and a north-south position-velocity diagram derived from these HI absorption observations. Comparing the sub-arcsec angular resolution HI absorption velocity field (Fig. 4c) with the centre of the $\sim 1$ '.7 angular resolution $\mathrm{CO}$ velocity field presented in Fig. $8 \mathrm{~b}$ of AHOO it is apparent that the "global" nuclear CO and HI dynamics are consistent. In both of these cases the general trend is for the gas to be rotating following a north-south velocity gradient of a few tens of $\mathrm{km} \mathrm{s}^{-1}$ per arcsec; further confirming the likely association of these two components.

On the scale of the OVRO CO emission the radio continuum peak is coincident with the kinematic centre of the galaxy. Centred close to this kinematic centre, these MERLIN observations provide a "close-up" view of the nuclear gas dynamics. In Fig. 4c the velocity field, although consistent with the CO, shows significant deviations and smaller scale velocity structure that is smoothed away by the larger $\mathrm{CO}$ synthesised beam. In particular the isovelocity contours of Fig. 4c show a significant twist toward the west as they cross the area of peak radio flux density (see also spectra 3 and 4 of Fig. 3). This twist in the velocity field is hinted at in the $\mathrm{CO}$ observations of AH00. However AH00 conclude that the gas in this region undergoes approximately solid-body rotation. This is broadly confirmed by these results, albeit only for a small cross-section through the rotating material, but it is apparent that the gas does also possess some additional non-circular motions superimposed upon this rotation.

As discussed in the previous section the HI absorption shows significant inhomogeneities in its column densities. In particular it is clear that the gas column densities are significantly lower against the bright radio continuum peak in the north of the source; along lines of sight where the gas is blueshifted relative to the galaxy's systemic velocity. Of course these column density variations may, in part, be the result of an underlying gas temperature gradient. However such an effect would need to be extreme to cause the large observed opacity differences and should not considered to be a dominant factor. Additionally AHOO find that the distribution of CO emitting gas is also asymmetric about the galaxy's kinematic centre. They observe bright, compact $\mathrm{CO}$ emission components redshifted relative to the systemic velocity of the galaxy and offset from the radio core by $\sim 1^{\prime \prime}$; no equivalent emission features are observed at similar blueshifted velocities.

Assuming that the radio continuum emission is related to the amount of recent star-formation occurring at anyone point; some of the areas with the most active ongoing star-formation will be located toward the brightest radio components. Further evidence from UV HST observations by Weistrop et al. (2004), where high concentration of young ( $\leq 20 \mathrm{Myr}$ old) star clusters are imaged, confirms that the majority of the recent starformation activity is situated toward the north and western parts of NGC 4194. Thus it appears that a considerable portion of the activity is situated on the blueshifted side of the source and away from the bulk of the cold gas.

Unlike emission experiments these absorption observations only detect gas that is in front of the source. Therefore if the star-formation areas to the north and west are being fed by the cold gas it is possible that the HI absorption column density toward the radio continuum peaks is depleted. Dynamically this might be considered as following the observed rotation velocity, where the gas approaches the rear of this area of starforming region, is partially converted to stars during its passage and hence has a reduced column density in front of this region. This scenario implies that the primary reservoir of gas that is fuelling these regions of starburst activity is in fact the gas toward the south of the radio continuum, where both the column densities of $\mathrm{HI}$ absorption and $\mathrm{CO}$ emission peak (i.e. an area along the same line-of-sight as the nuclear dust lane). Of course in this hypothesis it is equally plausible that the site of the active star-formation is moving toward the region with high gas density as the starburst evolves rather than the gas itself being transported into the stationary star-formation region.

\subsubsection{Physics of the cold gas}

As stated in Sect. 3.2 the column density of neutral hydrogen, $\mathrm{N}_{\mathrm{H}}$, is related to the gas spin (excitation) temperature, $T_{\text {spin }}$ and the observed HI absorption by

$N_{\mathrm{H}}=1.823 \times 10^{18} T_{\text {spin }} \int \tau \mathrm{d} V$ atoms $\mathrm{cm}^{-2}$

where $V$ is the in units of $\mathrm{km} \mathrm{s}^{-1}$ and $\tau$ is the measured opacity of the line. Thus any calculated values of $N_{\mathrm{H}}$ will be dependent upon the excitation temperature, $T_{\text {spin }}$.

The value of $T_{\text {spin }}$ for neutral hydrogen is controlled by three factors; pumping by Ly $\alpha$ photons, the absorption of $21 \mathrm{~cm}$ continuum radiation, and collisions (Field 1959a,b,c). It is well 
known that in high density regions that collisional excitation methods dominate; thus in these regions the spin temperature of HI will approach the gas kinetic temperature. For example measurements of HI emission and absorption column densities through dense regions of galactic disks are often consistent for excitation temperature in the range of a few 10 s to a few $100 \mathrm{~K}$ (e.g. Payne et al. 1983; Liszt et al. 1983; Braun 1997; Wolfire et al. 2003, and Refs. therein). It should be noted here that these calculations assume that the atomic gas, observed by either emission or absorption, is in a single component medium in equilibrium and thermalized (i.e. $n \gtrsim 1000 \mathrm{~cm}^{-3}$ ); see for example Kulkarni \& Heiles (1988). The assumption that $T_{\text {spin }}$ can be approximated to the gas kinetic temperature, $T_{\mathrm{k}}$, however, does not necessarily hold true especially (if a two component medium is assumed (a cold and warm neutral medium)) for areas of lower density gas clouds that are subject to irradiation by either significant levels Ly $\alpha$ photons or $21 \mathrm{~cm}$ continuum radiation (Wolfire et al. 2003). Work by Gallimore et al. (1999) further investigated these excitation processes addressing HI absorbing gas clouds within a few tens of parsecs of Seyfert nuclei. One of the primary conclusions of this work was that even in these regions where both an abundant $21 \mathrm{~cm}$ and Ly $\alpha$ source is present, $T_{\text {spin }}$ will asymptotically approach $T_{\mathrm{k}}$ for gas densities of $n \gtrsim 1000 \mathrm{~cm}^{-3}$ (i.e. a collision dominated regions). Whereas below this gas density the HI spin temperature, if in the presence of a $21 \mathrm{~cm}$ and/or Ly $\alpha$ photon source, will be significantly influenced by these other excitation processes resulting in $T_{\text {spin }}$ deviating away from the kinetic gas temperature (see Fig. 12 of Gallimore et al. 1999). However at this point we should also highlight the fact that for HI absorption, $\tau \propto\left(T_{\text {spin }} \Delta V\right)^{-1}$. Thus HI absorption studies, such as these, are most sensitive to the coldest atomic gas clouds.

Regarding the Medusa merger we have already assessed the plausibility of either an AGN embedded within the central starburst of this galaxy or whether the radio emission detected can be accounted for by star formation alone (see Sect. 4.3). From this discussion, although the presence of a weak or highly obscured active core cannot be eliminated, we conclude that the dominant energy input into the ISM of the Medusa is via the efficient starburst at its centre. Thus this ongoing star formation will be the primary heating processes for the ISM of NGC 4194.

The molecular ISM of the Medusa merger is characterized by an elevated $\mathrm{CO} /{ }^{13} \mathrm{CO} 1-0$ molecular line ratio of $19 \pm 4$ and relatively faint $\mathrm{HCN} 1-0$ emission $\left({ }^{12} \mathrm{CO} / \mathrm{HCN}>25\right)^{2}$. The faintness of $\mathrm{HCN}$ emission suggests that the average density in the molecular medium is below $10^{4} \mathrm{~cm}^{-3}$ and we suggest that the weak ${ }^{13} \mathrm{CO} 1-0$ line is caused by two effects: high gas temperatures $\left(T_{k}>50 \mathrm{~K}\right)$ and the presence of diffuse, unbound gas. High resolution observations reveal that the ${ }^{13} \mathrm{CO} 1-0$ line emission is relatively weak primarily in parts of the central dust lane and towards the bright central CO peak (Aalto et al. in prep.). While the latter is likely caused by elevated kinetic

\footnotetext{
${ }^{2}$ Recent observations by S. Aalto (unpublished) have detected very faint $\mathrm{HCN}$ emission from the compact, southern molecular peak which could be indicative of young or ongoing but obscured star formation in this region.
}

temperatures, the former is probably related to diffuse gas in a density-wave like situation, similar to that of the bar of NGC 7479 (Hüttemeister et al. 2000). In general most galaxies with elevated values of the $\mathrm{CO} /{ }^{13} \mathrm{CO} 1-0$ line ratio also have $f(60 \mu \mathrm{m} / f(100 \mu \mathrm{m}) \gtrsim 0.8$ indicating a high average dust temperature (Aalto et al. 1991, 1995). This further supports the notion that gas and dust is being heated by the ongoing starburst.

Considering these arguments it can be concluded that the molecular gas within the centre of the Medusa merger firstly exists in a relatively low density environment. In this sense, although there will clearly be higher density molecular gas clouds, the majority of the gas is in regions with $n \sim 1-5 \times 10^{3} \mathrm{~cm}^{-3}$. Additionally the molecular component appears to have slightly elevated kinetic temperatures $\left(T_{\mathrm{k}} \gtrsim\right.$ $50 \mathrm{~K}$ ). This provides us with an insight into the physics of the molecular clouds within the Medusa which can be translated into an upper limit for the density of the atomic gas and a lower limit on its kinetic temperature. These limits are valuable in that they help to define one extreme of the cold atomic gas phase within this merger. The primary caveat is that in most "cartoon" models of the ISM within galaxies the, coldest and densest, molecular clouds are cocooned within an envelope of more diffuse atomic gas which will be partially ionised. Thus, apart from the unlikely situation where the mixing of the atomic and molecular gas is complete, these molecular diagnostic tool can only be used to infer limits for the physics of the atomic gas phase.

\section{Conclusions}

Using radio continuum and $\mathrm{HI}$ absorption observations made with MERLIN we have studied the neutral hydrogen gas distribution and kinematics, in addition to the $1.4 \mathrm{GHz}$ radio continuum structure of the central kiloparsec of the Medusa merger at sub-arcsecond angular resolutions. These observations constitute the highest angular resolution study, to date, of either the neutral or molecular gas within this source.

Our highest resolution 1.4 GHz images of NGC 4194 reveal a pair of compact mJy radio components situated at the position of the peak in lower resolution radio images of this source. These two components are surrounded, primarily toward the south by a more diffuse region of weaker radio emission. These compact radio components are unresolved with sizes of $<25 \mathrm{pc}$ and a separation of $\sim 65 \mathrm{pc}$. We have used these radio observations to investigate the energy source of the radio emission and compare the two most probable scenarios; firstly that some portion of the radio emission is related to the presence of a weak AGN component in addition to the well known starburst emission from this source, or secondly, whether all of the radio emission can completely account for the extended nuclear starburst. Using a combination of the HI gas dynamics and starformation rates, derived from the nuclear radio emission compared, to those derived from observations at other wavelengths, we have tentatively concluded that the majority, if not all, of the emission is related to star-formation activity. At present we cannot categorically eliminate the possibility of a weak AGN component without further observational studies such as 
further high sensitivity multi-frequency VLBI observation or high resolution X-ray observations.

By observing HI via absorption we have been able to trace the gas dynamics in front of the background radio continuum within the half central kiloparsec of the Medusa merger. The distribution of the absorbing HI gas shows a significant enhancements toward the south of the radio continuum source. This area is along the same line-of-sight as the peaks in the ${ }^{12} \mathrm{CO}$ emission observed by AHOO and is also co-spatial with a cross-nuclear dust lane (see Fig. 1). Following the results of AHOO we conclude that all three of these cold ISM components (dust, $\mathrm{CO}$ and atomic hydrogen) are probably physically related, with both the $\mathrm{CO}$ emission and $\mathrm{HI}$ absorption exhibiting similar dynamics. The HI absorption traces a relatively shallow, approximately, north-south velocity gradient of $\sim 320 \mathrm{~km} \mathrm{~s}^{-1} \mathrm{kpc}^{-1}$ over $\sim \frac{1}{2} \mathrm{kpc}$ of the background radio continuum. This gradient is consistent with that observed in $\mathrm{CO}$ by AH00. Considering this gradient and the distribution of the absorbing $\mathrm{HI}$ and the most active star-forming regions we discuss the possible role of the gas dynamics with respect to the fuelling of the starburst. Since the Medusa merger has been extensively studied via a variety of molecular gas transitions we have also discussed the use of these as diagnostic tools to infer some limits on the physical environment that the molecular and atomic gas resides in.

Acknowledgements. R.J.B. would like to acknowledge financial support by the European Commission's I3 Programme "RADIONET" under contract No. 505818. We would like to thank all the MERLIN staff for their assistance with these observations. In addition we thank Susan Neff for kindly sharing with us her recent VLA observations of NGC 4194 prior to publication. MERLIN is a national facility operated by the University of Manchester on behalf of PPARC in the UK. The authors wish to thank the Canadian Astronomy Data Centre, which is operated by the Hertzberg Institute of Astrophysics, National Research Council of Canada for providing recalibrated HST data to the author as a guest user.

\section{References}

Aalto, S., Johansson, L. E. B., Booth, R. S., \& Black, J. H. 1991, A\&A, 249,323

Aalto, S., Booth, R. S., \& Black, J. H., \& Johansson, L. E. B. 1995, A\&A, 300, 369

Aalto, S. \& Hüttemeister, S. 2000, A\&A, 362, 42 (AH00)

Aalto, S., Hüttemeister, S., \& Polatidis, A. G. 2001, A\&A, 372, L29

Armus, L., Heckman, T. M., \& Miley, G. K. 1990, ApJ, 364, 471

Beswick, R. J., Pedlar, A., Mundell, C. G., \& Gallimore, J. F. 2001, MNRAS, 325, 151

Beswick, R. J., Pedlar, A., \& Holloway, A. J. 2002, MNRAS, 329, 620

Beswick, R. J., Pedlar, A., Clemens, M. S., \& Alexander, P. 2003, MNRAS, 346, 424
Beswick, R. J., Peck, A. B., Taylor, G. B., \& Giovannini, G. 2004, MNRAS, 352, 49

Braun, R. 1997, ApJ, 484, 637

Cole, G. H. J., Pedlar, A., Mundell, C. G., Gallimore, J. F., \& Holloway, A. J. 1998, MNRAS, 301, 782

Condon, J. J., Helou, G., Sanders, D. B., \& Soifer, B. T. 1990, ApJS, 73,359

Condon, J. J. 1992, ARA\&A, 30, 575

Cram, L., Hopkins, A., Mobasher, B., \& Rowan-Robinson, M. 1998, ApJ, 507, 155

Field, G. B. 1959a, ApJ, 129, 525

Field, G. B. 1959b, ApJ, 129, 536

Field, G. B. 1959c, ApJ, 129, 551

Gallimore, J. F., \& Beswick, R. J. 2004, AJ, 127, 329

Gallimore, J. F., Baum, S. A., O’Dea, C. P., Pedlar, A., \& Brinks, E. 1999, ApJ, 524, 684

Genzel, R., Lutz, D., Sturm, E., et al. 1998, ApJ, 498, 579

Haarsma, D. B., Partridge, R. B., Windhorst, R. A., \& Richards, E. A. 2000, ApJ, 544, 641

Heckman, T. M., Smith, E. P., Baum, S. A., et al. 1986, ApJ, 311, 526

Högbom, J. A. 1974, A\&AS, 15, 417

Hopkins, A. M., Schulte-Ladbeck, R. E., \& Drozdovsky, I. O. 2002, ApJ, 124, 862

Hüttemeister, S., Aalto, S., Das, M., \& Wall, W. F. 2000, A\&A, 363, $93 \mathrm{H}$

Jackson, N., Beswick, R. J., Pedlar, A., et al. 2003, MNRAS, 338, 643

Kewley, L. J., Heisler, C. A., Dopita, M. A., et al. 2000, ApJ, 530, 704

Kulkarni, S. R., \& Heiles, C. 1988, in Galactic and Extragalactic Radio Astronomy, ed. G. L. Verschuur, \& K. I. Kellemann (Berlin: Springer), 95

Liszt, H. S., van der Hulst, J. M., Burton, W. B., \& Ondrechen, M. P. 1983, A\&A, 126, 40

Liu, C. T., \& Kennicutt, R. C., Jr. 1995, ApJS, 100, 325

Lonsdale, C. J., Lonsdale, C. J., \& Smith, H. E. 1992, ApJ, 391, 629

Maloney, P. R., Hollenbach, D. J., \& Tielens, A. G. G. M. 1996, ApJ, $466,561 \mathrm{M}$

Muxlow, T. W. B., Pedlar, A., Wilkinson, P. N., et al. 1994, MNRAS, 266, 455

Neff, S. G., Campion, S. D., \& Ulvestad, J. S. 2001, AAS, 199, 8705

Payne, H. E., Salpeter, E. E., \& Terzian, Y. 1983, ApJ, 272, 540

Prestwich, A. H., Joseph, R. D., \& Wright, G. S. 1994, ApJ, 422, 73

Staveley-Smith, L., \& Davies, R. D. 1987, MNRAS, 224, 953 S

Storchi-Bergmann, Th., Calzetti, D., \& Kinney, A. 1994, ApJ, 429, 572

Thean, A., Pedlar, A., Kukula, M. J., Baum, S. A., \& O’Dea, C. P. 2001, MNRAS, 325, 737

Thomasson, P. 1986, QJRAS, 27, 413

Ulvestad, J. S., Wilson, A. S., \& Sramek, R. A. 1981, ApJ, 247, 419

van Breugel, W. J. W., Schilizzi, R. T., Hummel, E., \& Kapahi, V. K. 1981, A\&A, 96, 310

Weistrop, D., Eggers, D., Hanock, M., et al. 2004, AJ, 127, 1360

Wills, K. A., Pedlar, A., \& Muxlow, T. W. B. 1998, MNRAS, 298, 347

Wolfire, W. G., McKee, C. F., Hollenbach, D., \& Tielens, A. G. G. M. 2003, ApJ, 587, 278 